\title{
An Effective Gear Fault Diagnosis Method Based on Singular Value Decomposition and Frequency Slice Wavelet Transform
}

\author{
Fu-Cheng Zhou, Gui-Ji Tang, and Yu-Ling He \\ Institute of Equipment Fault Diagnosis and Testing Technology, North China Electric Power University, Baoding, Hebei, China \\ Correspondence should be addressed to Yu-Ling He; heyuling1@163.com
}

Received 29 June 2016; Accepted 15 September 2016

Academic Editor: Zuohua Huang

Copyright ( 2016 Fu-Cheng Zhou et al. This is an open access article distributed under the Creative Commons Attribution License, which permits unrestricted use, distribution, and reproduction in any medium, provided the original work is properly cited.

\begin{abstract}
The ability of the frequency slice wavelet transform (FSWT) to distinguish the fault feature is weak under the condition of strong background noise; in order to solve this problem, a fault feature extraction method combining the singular value decomposition (SVD) and FSWT was proposed. Firstly, the Hankel matrix was constructed using SVD, based on which the SVD order was determined according to the principle of the single side maximum value. Then, the denoised signal was further processed by the FSWT to obtain the time-frequency spectrum of the passband. Finally, the detailed analysis was carried out in the time-frequency area with concentrated energy, and the signal was reconstructed by the inverse-FSWT. The processing effect for the pitting corrosion and the tooth broken faults of the gears shows that the faulty feature can be extracted effectively from the envelope spectrum of the reconstructed signal, which means the proposed method is able to help obtain a qualified result and has the potential to be carried out for the practical engineering application.
\end{abstract}

\section{Introduction}

Gears are important transmission parts for rotating machines. It is shown that about $60 \%$ of failures of gearbox are caused due to gear faults. Therefore, gear fault diagnosis is of great significance. Typically, the amplitude modulation components shown in the vibration signal, which are produced by the gear engagement frequency and its $n$th harmonics, indicate the fault features. Usually, the fault feature is interfered by the noise, resulting in the difficulties to extract and recognize the signal effectively. Many scholars have put forward a lot of methods, such as wavelet transform (WT) and short time Fourier transform, which have achieved satisfactory results. But, during the nonlinear and nonstationary gear fault signal processing, it is difficult to select the base function for WT [1]. For the short time Fourier transform which is restricted to the principle of the Heisenberg uncertainty, the timefrequency resolution cannot achieve the best results [2], while for the Wigner-Ville distribution it has the inherent crossterm interference problems. Yan proposed the frequency slice wavelet transform (FSWT) by introducing the frequency section function based on WT $[3,4]$, which utilizes traditional Fourier transform to obtain the function of the time-frequency analysis for signal filtering and division flexibly. In view of the advantages of FSWT in signal analysis and processing, scholars have applied this method to the field of mechanical fault diagnosis. For example, Duan et al. used FSWT to diagnose the friction fault of the refinery gearbox and also the rub-impact fault of the turbine rotor $[5,6]$. Zhong et al. applied morphological filtering based FSWT to the bearing fault diagnosis [7].

The mechanical fault signal processing method based on FSWT can obtain a certain result in actual diagnosis application. However, during the research, the authors found that the fault feature extraction result using FSWT is still not ideal under the condition of strong noise interference. Thus, it is significant to reduce the noise in the original signal. The information signal matrix can be decomposed into a series of singular value matrixes and singular value vectors in the subspace by the nonlinear filtering method singular value decomposition (SVD) [8]. And the noise components in the mixed signal can be eliminated [9]. Thus, the SVD method has a certain advantage in extracting the fault signal [1013]. In order to extract and separate the gear fault feature effectively, a fault feature extraction method by combining SVD with FSWT was proposed in this paper to diagnose the 
pitting and the tooth broken faults of gears. The reliability of this method was verified by the test data.

\section{Frequency Slice Wavelet Transform Analysis}

2.1. Frequency Slice Wavelet Transform. For any signal $f(t) \in$ $L^{2}(R)$, its frequency slice wavelet transform is $[3,4]$

$$
W(t, \omega, \lambda, \sigma)=\frac{1}{2 \pi} \lambda \int_{-\infty}^{+\infty} \widehat{f}(u) \widehat{p}^{*}\left(\frac{u-\omega}{\sigma}\right) e^{i u t} d u,
$$

where $\sigma$ is the scale factor $(\sigma \neq 0)$ and $\lambda$ is the energy coefficient $(\lambda \neq 0)$. They are either constant or the functions of $\omega$ and $t . \widehat{p}(\omega)$ is the Fourier transform of $p(t)$, while $\widehat{p}^{*}(\omega)$ is the conjugate function of $\widehat{p}(\omega)$. $\widehat{p}(\omega)$ is called frequency slice function (FSF), and (1) can be translated into its timedomain format by the Parseval equation $[3,4]$

$$
\begin{aligned}
& W(t, \omega, \lambda, \sigma) \\
& \quad=\sigma \lambda e^{i \omega t} \int_{-\infty}^{+\infty} f(\tau) e^{-i \omega t} p^{*}[\sigma(\tau-t)] d \tau .
\end{aligned}
$$

2.2. Scale Selection of Frequency Slice Wavelet Transform. Assume that $\lambda=1$; according to the principle of Morlet transform, keeping $\sigma \propto \omega$ is a good choice. Assume that $\sigma=\omega / k(k>0)$, and

$$
W(t, \omega, \lambda, k)=\frac{1}{2 \pi} \int_{-\infty}^{+\infty} \widehat{f}(u) \hat{p}^{*}\left[k \frac{(u-\omega)}{\omega}\right] e^{i u t} d u,
$$

where $k$ is the time-frequency resolution coefficient. The insertion of the factor $k((u-\omega) / \omega)$ in the window function can be adjusted to tune the transform to be more sensitive to the frequency and the time. Then, (2) can be changed into

$$
W(t, \omega, k)=\frac{1}{k} \omega e^{i \omega t} \int_{-\infty}^{+\infty} f(\tau) e^{i \omega t} p^{*}\left[\frac{\omega(t-\tau)}{k}\right] d \tau .
$$

Due to the Heisenberg uncertainty principle, it is needed to reach a compromise between the time and the frequency resolution. There are two eclectic parameters defined to estimate the scales $s$ and $k$. The first one is the frequency resolution ratio of the signal and recorded as $\eta(\eta=$ $\Delta \omega / \omega)$. The second one is the expected response ratio of the amplitude, and it is shown as $v(0<v \leq 1)$.

If $(t)=e^{i \omega_{0} t}$ and its FSWT satisfies that $\mid W\left(t, \omega_{0}+\right.$ $\Delta \omega, \lambda, \sigma)|/| W\left(t, \omega_{0}, \lambda, \sigma\right) \mid \leq v$, then

$$
|\widehat{p}(k \eta)| \leq v|\widehat{p}(0)| \text {. }
$$

If $(t)=\delta\left(t-t_{0}\right)$ and its FSWT satisfies $\mid W\left(t_{0}+\right.$ $\Delta t, \omega, \lambda, \sigma)|/| W\left(t_{0}, \omega, \lambda, \sigma\right) \mid \leq v$, then

$$
\left|p\left(\frac{\mu}{k \eta}\right)\right| \leq v|p(0)|,
$$

where $\mu=\Delta \omega \Delta t$.

Specially, let FSF be the Gaussian function $\widehat{p}(\omega)=e^{-\omega^{2} / 2}$, and then $\mu=1 / 2$. According to inequalities (5) and (6), we have

$$
\frac{\sqrt{2 \ln (1 / v)}}{\eta} \leq k \leq \frac{\mu}{\eta \sqrt{2 \ln (1 / v)}} .
$$

If inequalities (6) and (7) are satisfied simultaneously in the time-frequency domain, then they have a unique solution, $v=e^{-\mu / 2}, k=\sqrt{2} / 2 \eta$.

2.3. Inverse Transform. FSWT is used to decompose the signal in the time-frequency domain. Generally, it is necessary that FSWT should be reversible for signal filtering and segmenting. The original signal $f(t)$ can be reconstructed by

$$
f(t)=\frac{1}{2 \pi \lambda} \int_{-\infty}^{+\infty} \int_{-\infty}^{+\infty} W(t, \omega, \lambda, \sigma) e^{i \omega(t-\tau)} d \tau d \omega .
$$

\section{Singular Value Decomposition Principle}

The Hankel matrix of the practical sampling signal $X=$ $\left(x_{1}, x_{2}, \ldots, x_{N}\right)$ can be constructed as

$$
A=\left[\begin{array}{cccc}
x_{1} & x_{2} & \cdots & x_{n} \\
x_{2} & x_{3} & \cdots & x_{n+1} \\
\vdots & \vdots & \ddots & \vdots \\
x_{m} & x_{m+1} & \cdots & x_{m+n-1}
\end{array}\right],
$$

where $N=m+n-1, m \geq n$. The singular value decomposition can be expressed as

$$
A=U \Sigma V^{T}
$$

where $U$ is $m \times m$ orthogonal matrix that satisfies $U U^{T}=I(I$ is a unit matrix), $V$ is orthogonal matrix that satisfies $V V^{T}=$ $I$, and $\Sigma=\operatorname{diag}\left(\sigma_{1}, \sigma_{2}, \ldots, \sigma_{n}\right)$ is the diagonal matrix whose elements are the singular values of matrix $A$.

The useful signal can be reflected by the larger singular values, while the noise signal can be determined by the left smaller singular values. The noise in the source signal will be removed when the small singular value is deleted. Then the matrix $\widehat{A}$, which is the best approximation matrix of $A$ and with the rank of $r$, can be obtained by means of the inverse calculus process of SVD. Further, the antidiagonal elements of the matrix $\widehat{A}$ are added together, so that the denoised signal $\widehat{A}_{s}$ can be obtained:

$$
\widehat{A}_{s}=\sum_{i=1}^{r} \sigma_{i} u_{i} v_{i}^{T}
$$

The differential spectrum sequence $b_{i}=\sigma_{i}-\sigma_{i+1}(i=$ $1,2, \ldots, n-1)$ of the singular values describes the specific changes of the singular value sequence. The constructed differential sequence $B=\left(b_{1}, b_{2}, \ldots, b_{n-1}\right)$ reflects the change of the two adjacent singular values. According to the definition of the differential spectrum sequence, the greater the difference value of the two adjacent singular values is, the more obvious the features reflected by the differential spectrum will be. The gap of the response before and after peak one is not the degree of the correlation between the noise and the useful signal, so the effective singular value of the appropriate rank order is chosen to strip the noise from 


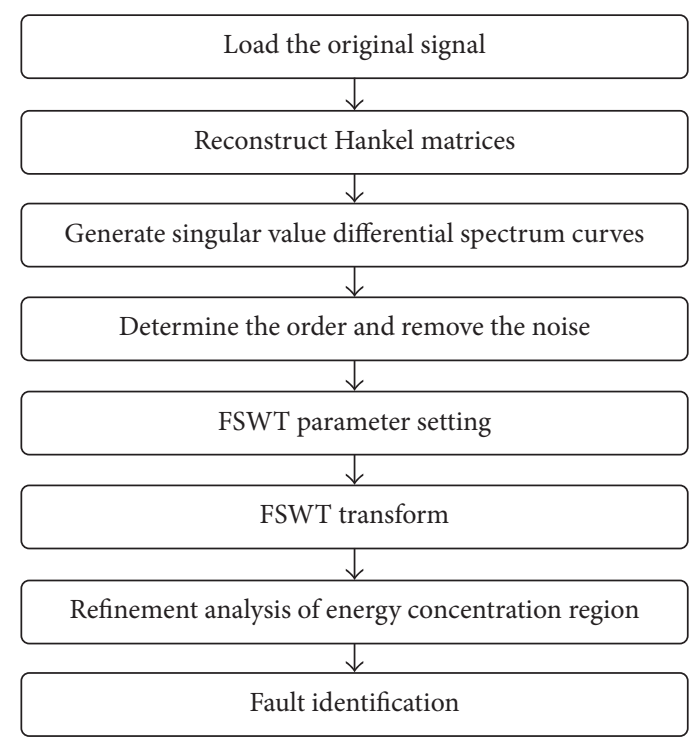

FIGURE 1: Flow chart of signal processing.

the useful signal effectively. This paper adopts the unilateral maximum principle proposed in [13] and selects the first unilateral adjacent peak in the singular value differential spectrum from right to left to determine the signal reconstruction efficiency rank order. This efficiency rank order can be obtained by finding the largest absolute difference value between the two adjacent points in the differential spectrum. Finally, reconstruct the useful signal and eliminate the noise effectively.

\section{Time-Frequency Analysis Method Based on SVD and FSWT}

In order to denoise and reconstruct the signal, the paper combines SVD with FSWT to diagnose the gear fault. The flow chart of the proposed method is shown in Figure 1, and the main steps are as follows:

(1) The original signal $x(t)$ is reconstructed by Hankel matrices in the phase space, and the first peak to the greatest change point, which is also the rank order of reconstructed signal, is selected from right to left. According to the change of the singular value differential spectrum, the noise can be removed and the useful signal can be extracted.

(2) Select the appropriate frequency slice function $\widehat{p}(\omega)$, then estimate the frequency resolution ratio $\eta$ and the amplitude response ratio, and calculate the timefrequency resolution coefficient $k$.

(3) Select the frequency slice interval within the Nyquist analysis frequency band, and the time-frequency decomposition coefficient $W(t, \omega, k)$ of the signal can be obtained via FSWT. Then the time-frequency diagram of the signal in the whole frequency band can be obtained.

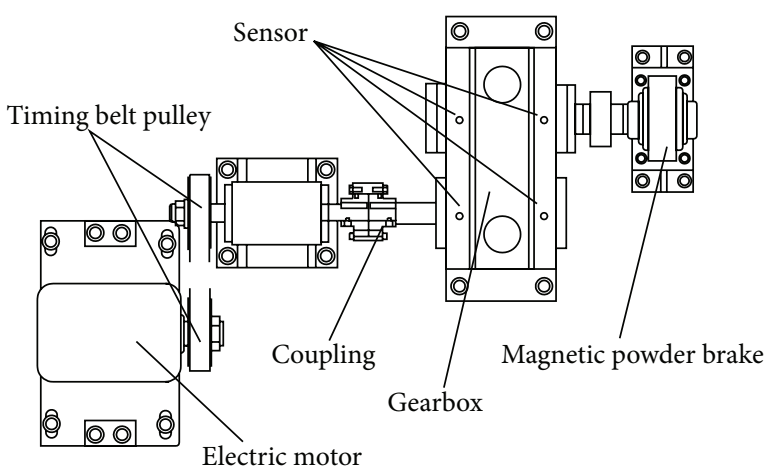

FIGURE 2: Experimental platform.

(4) Analyze the signal distribution area in the timefrequency diagram, and the signal frequency slice refinement analysis is carried out according to the signal energy concentration range. Finally, the fault features can be extracted through decomposing and reconstructing the signal.

\section{Application Examples}

5.1. Pitting Corrosion Fault. A tiny dent is machined to simulate the gear pitting fault on the gear tooth surface, and the structure diagram of test platform is showed in Figure 2. Four acceleration sensors are arranged in the gearbox cover nearing the bearing. The sampling frequency is $5120 \mathrm{~Hz}$, and the sampling points are 4096 points. The motor speed is $825 \mathrm{rpm}$. The number of the driving gear teeth is 55 , while the tooth number of the gear on the output shaft is 75 . The rotating frequency of the input shaft is $f_{i}=13.75 \mathrm{~Hz}$, while the rotating frequency of the output shaft is $f_{o}=10.1 \mathrm{~Hz}$.

The waveform and the spectrum of the tested signal are shown in Figure 3. There are little impacts appearing in the time-domain waveform, but the time interval is not obvious. The gear meshing frequency, $757 \mathrm{~Hz}$, is very prominent in the spectrum, and the edge band component appears on both sides. This indicates the occurrence of the signal modulation which causes the side band interval (fault frequency) to be less than the resolution. Consequently, the gear fault cannot be distinguished. The envelope spectrum analysis is performed on the tested signal, and the result is shown in Figure 4. It can be found that the output shaft frequency $f_{o}$ and its multiplication frequencies are prominent. Meanwhile, the input shaft frequency $f_{i}$ and its double frequency $2 f_{i}$ are also prominent. This brings about great difficulty in judging whether the driving gear or the driven gear is faulted. Thus, the traditional envelope analysis method for this fault is not qualified.

For the sake of comparison, we used the method of FSWT to analyze the tested signal directly. According to (7), $k=$ $\sqrt{2 \ln (1 / v)} / \eta$. Usually, scholars set $v=\sqrt{2} / 2$ and $\eta=0.025$ $[3,4]$. Then $k=33.3$, and the parameters of FSWT are $\widehat{p}(\omega)=$ $e^{-\omega^{2} / 2}, \lambda=1$, and $\sigma=\omega / k$. The frequency slice interval is $\left[\begin{array}{ll}0 & 2560 \mathrm{~Hz}\end{array}\right]$. Figure 7(a) is the result of the signal proposed by FSWT. It can be seen from the figure that the distribution 


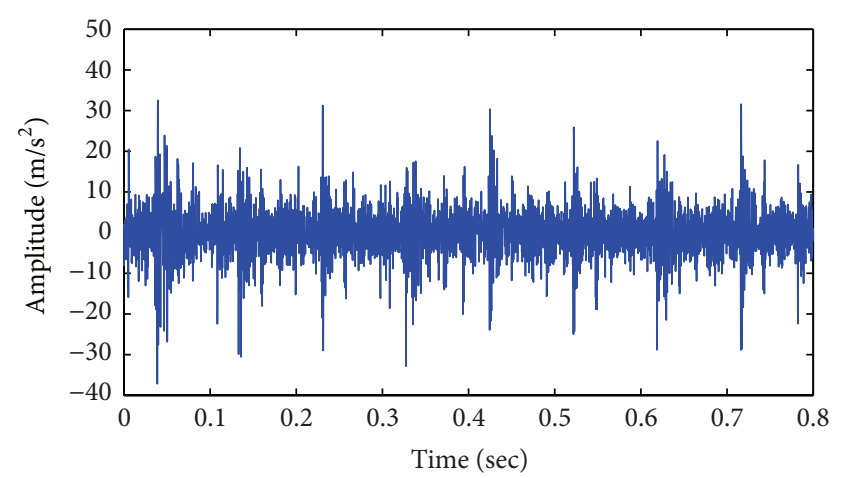

(a) Waveform of tested signal

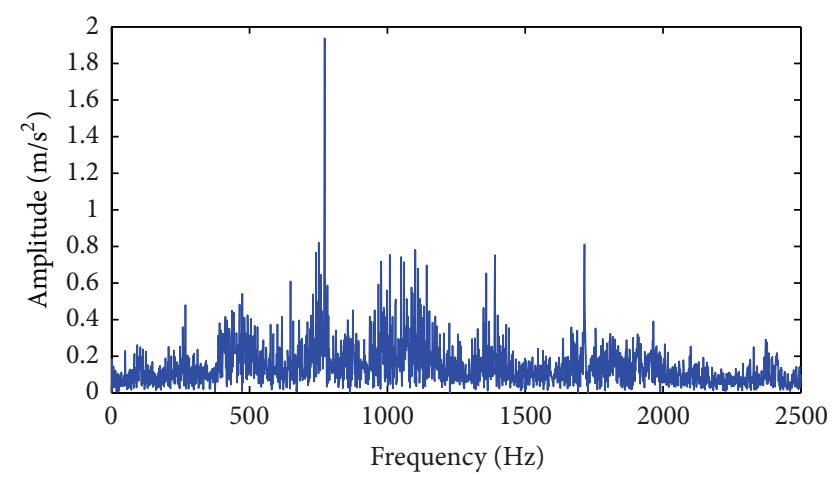

(b) Spectrum of tested signal

FIGURE 3: Waveform and spectrum of tested signal.

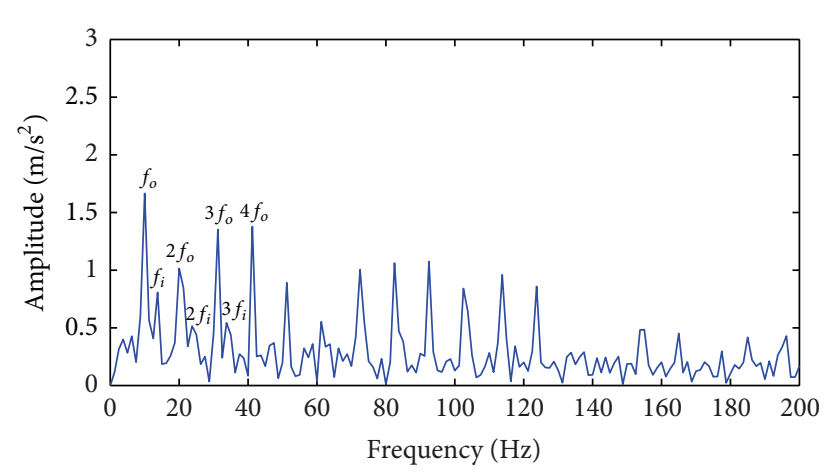

FIGURE 4: Envelope spectrum of tested signal.

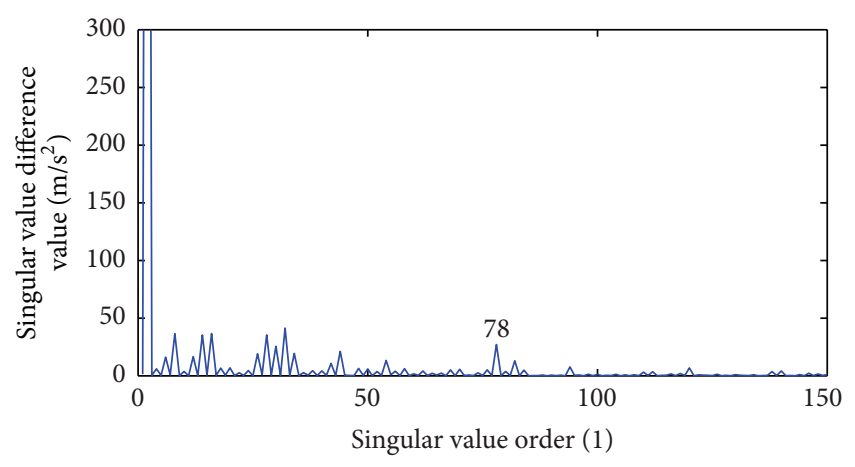

Figure 5: Singular value differential spectrum.

of the whole signal has no obvious regularity on the frequency axis. Consequently, it can not refine the frequency partition of the signal. This means FSWT can not extract the fault characteristics effectively.

Then we used the method proposed in this paper to analyze the tested signal. Firstly, the original signal is processed via SVD, and the result is indicated in Figure 5. It is shown that, from right to left, the gap between the two peaks of Point 77 and Point 78 is the biggest. Secondly, this point is selected as the effective rank order of the reconstructed signal to reduce the noise. Finally, the denoised signal is reconstructed,

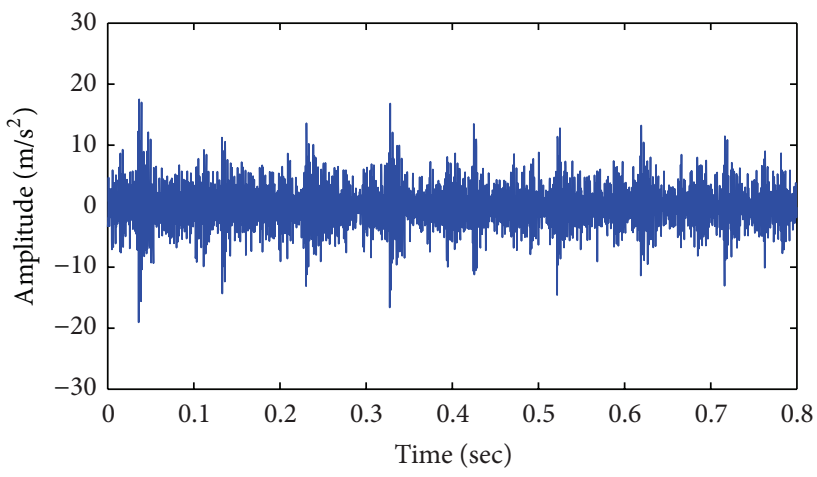

FIgURE 6: The waveform of the signal after noise reduction.

and the obtained signal waveform is shown in Figure 6. In this figure, only small impulse can be observed.

The time-frequency diagram is shown in Figure 7(b) after noise reduction, repeating the various parameters of FSWT directly. In this figure, there is a component in the vicinity of the gear meshing frequency at $750 \mathrm{~Hz}$, whose frequency range is narrow but duration is long. This indicates the occurrence of amplitude modulation. A series of equal interval and wide range components are distributed in the range of $850-1300 \mathrm{~Hz}$, while the time-frequency distribution is not outstanding in other frequency ranges. The detailed FSWT analysis was carried out for the frequency band of $850-1300 \mathrm{~Hz}$, and the time-frequency diagram is shown in Figure 8. It can be found that the component has the features of wide frequency band and short duration, which is the typical characteristic of shock response. These components, which have the obvious amplitude modulation effect, can be seen from the reconstructed signals in Figure 9. Then, the envelope analysis is performed on the reconstructed signal. In Figure 10, it can be found that the modulation frequency is about $10 \mathrm{~Hz}$, and its double frequency is also clearly visible. These characteristics show that the tested signal contains a periodic impact component with the frequency of $10 \mathrm{~Hz}$, that is, the switch frequency of the output shaft. The analysis results are consistent with the actual situation, and the fault feature is extracted successfully. 


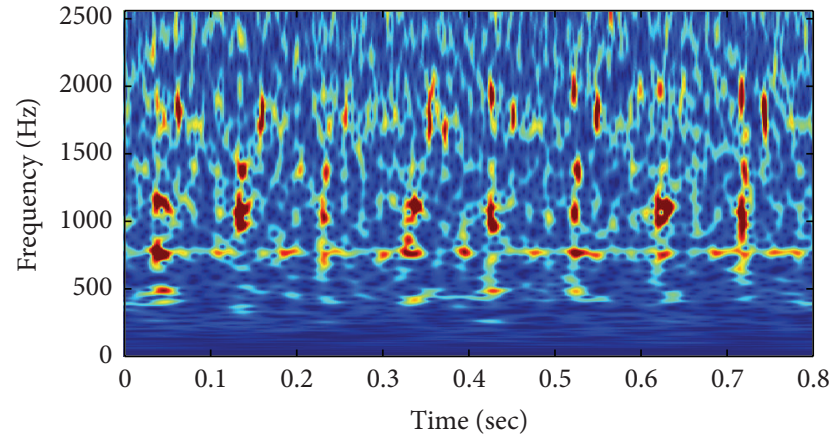

(a) Time-frequency distribution before denoising

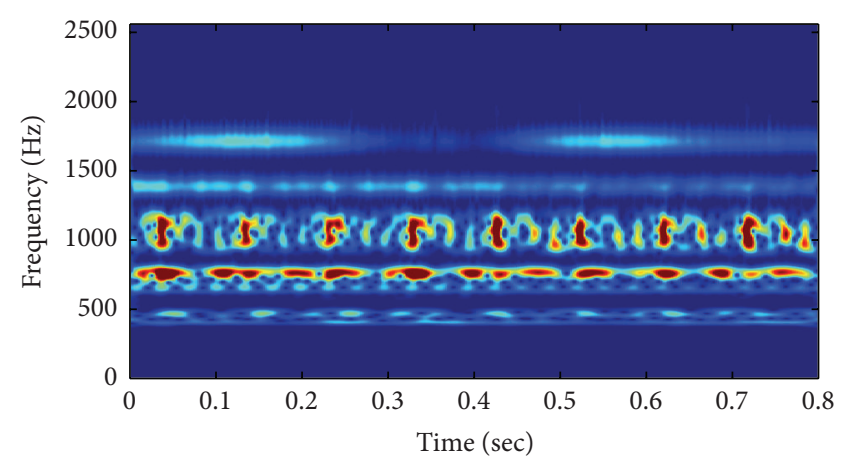

(b) Time-frequency distribution after denoising

FIgURE 7: Time-frequency distribution of the vibration signal.

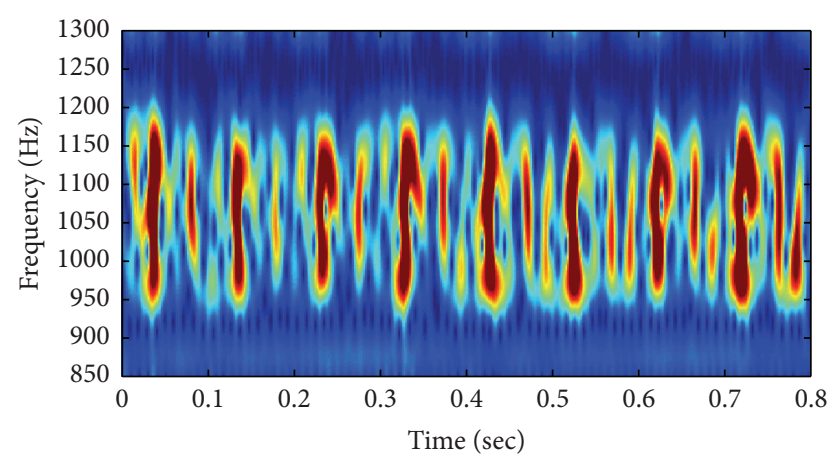

FIGURE 8: FSWT time-frequency distribution over the band of 850$1300 \mathrm{~Hz}$.

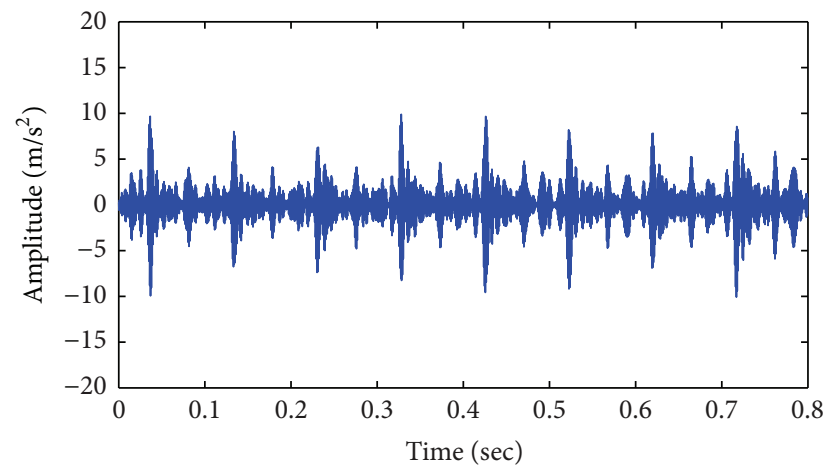

Figure 9: Reconstructed signal over the band of $850-1300 \mathrm{~Hz}$.

5.2. Tooth Broken Fault. The tooth broken fault is set on the input shaft pinion, and the length of the tooth breakage is about a quarter of the tooth width. The number of the teeth on the input shaft is 55, while the number of the teeth on the output shaft is 75 . The input shaft speed is $878 \mathrm{rpm}$, and the rotating frequency of the input shaft $f_{i}=14.6 \mathrm{~Hz}$, while the rotating frequency of the output shaft $f_{o}=10.7 \mathrm{~Hz}$. The mesh frequency of the gears $f_{c}=805 \mathrm{~Hz}$. The time-domain waveform and the spectrum of the vibration signal are shown in Figure 11.

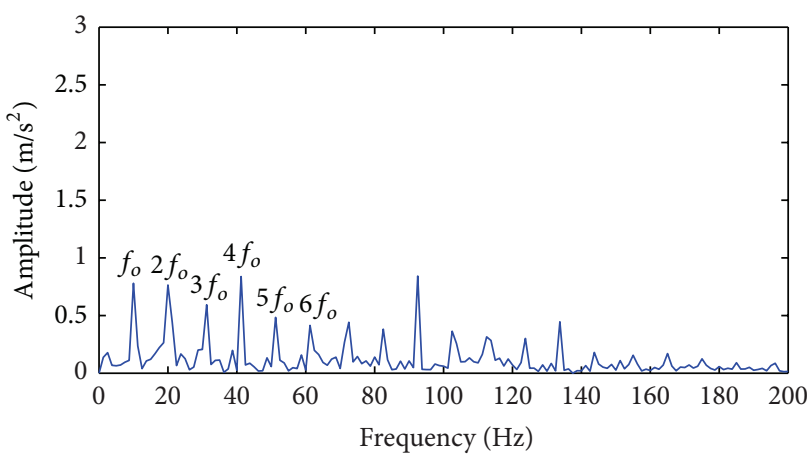

FIGURE 10: Envelope spectrum of reconstructed signal after FSWT.

It is shown that the impact phenomenon is relatively obvious in the waveform of the tested signal. However, there is no apparent distribution regularity. It can also be observed that though there are some prominent lines in the spectrum, they are neither the meshing frequency nor the harmonic frequencies of the meshing. The meshing frequency is submerged by the strong noise. In the meantime, the side band of the meshing frequency from the low frequency to the high frequency still exists. The large amplitude and the wide distribution show that the modulation phenomenon is obvious. So it is unable to identify the fault using the timedomain waveform and the spectrum diagram.

The envelope demodulation is performed on the vibration signal in the low frequency band and the result is shown in Figure 12. It shows that the double frequency $2 f_{i}\left(f_{i}\right.$ is the rotating frequency of the input shaft) is very outstanding. However, the fundamental frequency and the other multiplication frequencies are not obvious. Therefore, it is hard to judge the fault of the gear.

In a further study, FSWT was employed to the original signal directly, taking the frequency slice function $\widehat{p}(\omega)=$ $e^{-\omega^{2} / 2}$, and the parameters $\lambda=1, \eta=0.025$. The timefrequency diagram is shown in Figure 15(a). It is suggested from the figure that there is no obvious law of the signal distribution, which means the signal can not be clearly divided. So it is difficult to extract the features from the faulty signal. 


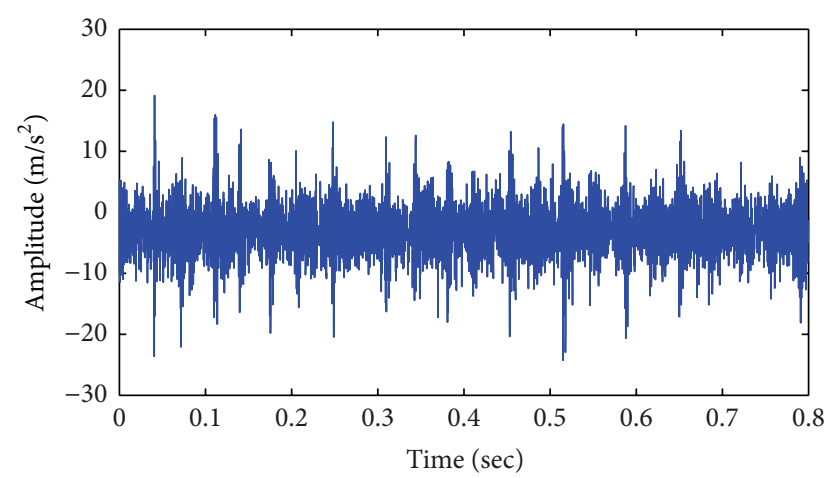

(a) Waveform of tested signal

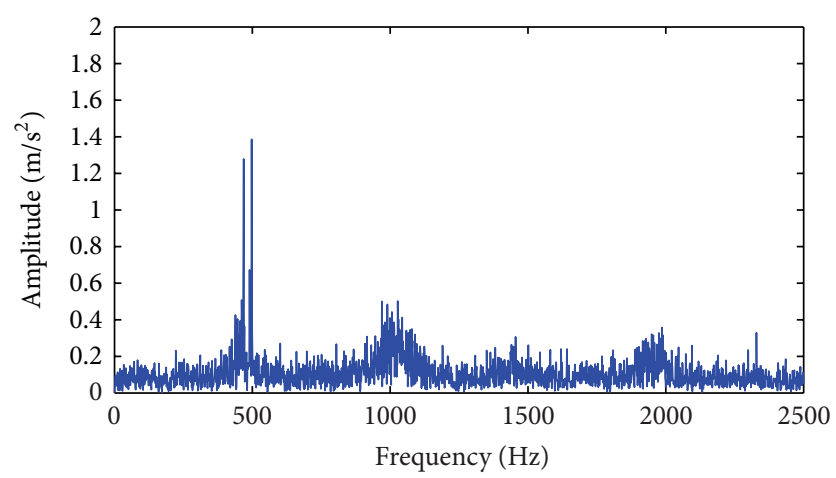

(b) Spectrum of tested signal

FIGURE 11: Waveform and spectrum of tested signal.

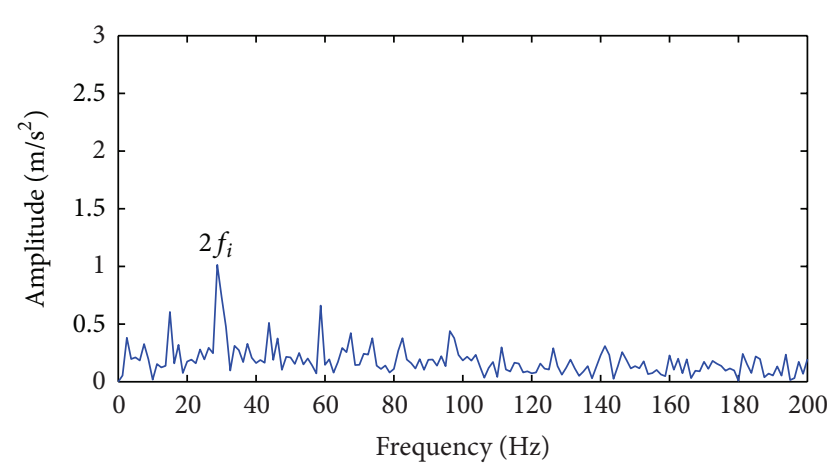

FIGURE 12: Envelope spectrum of tested signal.

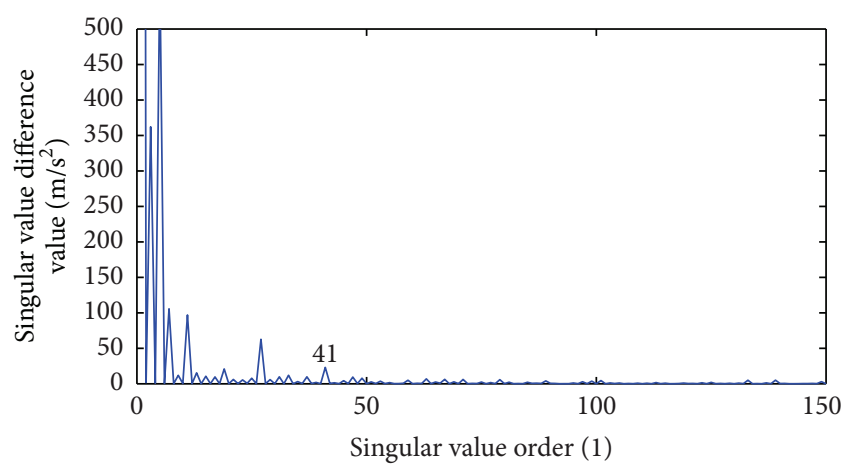

FIGURE 13: Singular value differential spectrum.

In order to reduce noise, singular value decomposition (SVD) was performed on the vibration signal, as indicated in Figure 13. It can be seen that the gap between the Point 41 and its adjacent left peak is the biggest. This point is chosen as the effective rank order of the reconstructed signal to reduce noise, and the waveform of the denoised signal is shown in Figure 14.

After frequency slice analysis, the time-frequency diagram is shown in Figure 15(b). It can be observed in this figure that a series of signal components are distributed trimly in the frequency band of $900-1200 \mathrm{~Hz}$, while in other bands the signal components are not prominent. Slice

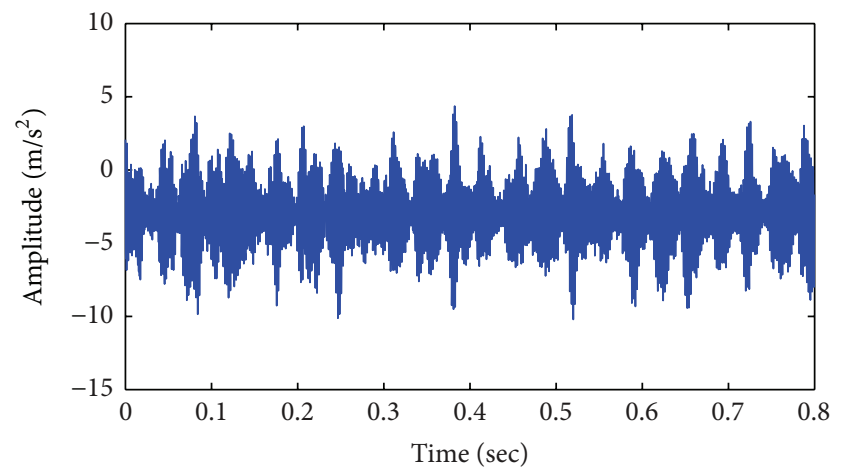

FIGURE 14: The waveform of the signal after noise reduction.

refinement analysis is carried out in the frequency band of $900-1200 \mathrm{~Hz}$, as indicated in Figure 16. It can be seen that these banded components have the features of wide frequency range and short duration, which imply the shock response characteristics of the signal. Figure 17 shows the reconstructed signal, where the components have obvious amplitude modulation effects. The envelope demodulation is performed on the reconstructed signal, as indicated in Figure 18. It shows that the modulation frequencies are the rotating frequency of the input shaft and its higher-order harmonics. This means the driving gear (the smaller gear) on the input shaft has local faults. This result is consistent with the actual situation.

\section{Conclusion}

This paper proposed a vibration signal processing method based on singular value decomposition (SVD) and frequency slice wavelet transform (FSWT). The primary conclusions drawn from the study are as follows:

(1) The fault characteristic component is not enough in the tested gear vibration signal because it is affected by the transmission path and the background noise. Thus, it is relatively difficult to extract the fault feature, and the traditional envelope demodulation method is usually less effective. The proposed method 


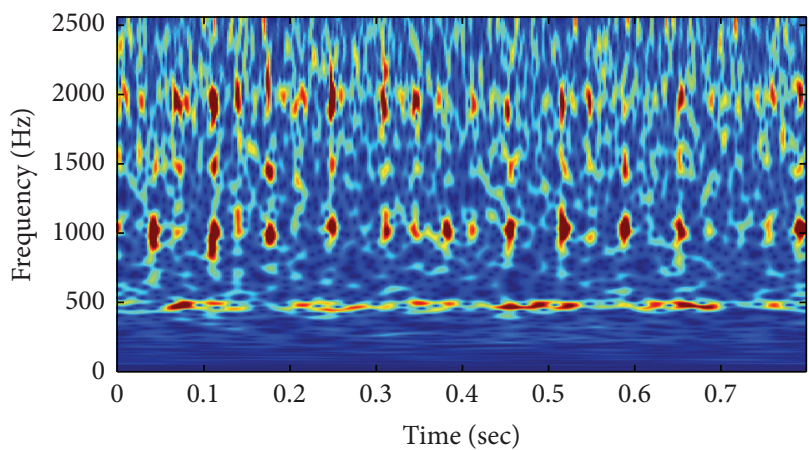

(a) Time-frequency distribution before denoising

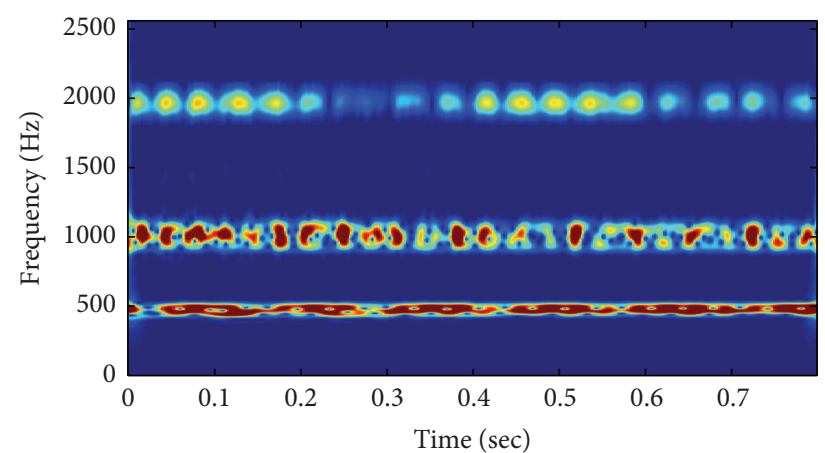

(b) Time-frequency distribution after denoising

FIGURE 15: Time-frequency distribution of the vibration signal.

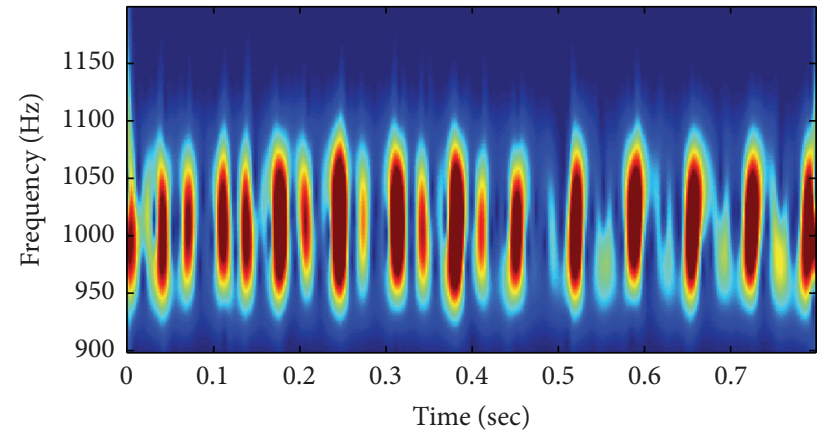

FIGURE 16: FSWT time-frequency distribution over the band 900$1200 \mathrm{~Hz}$.

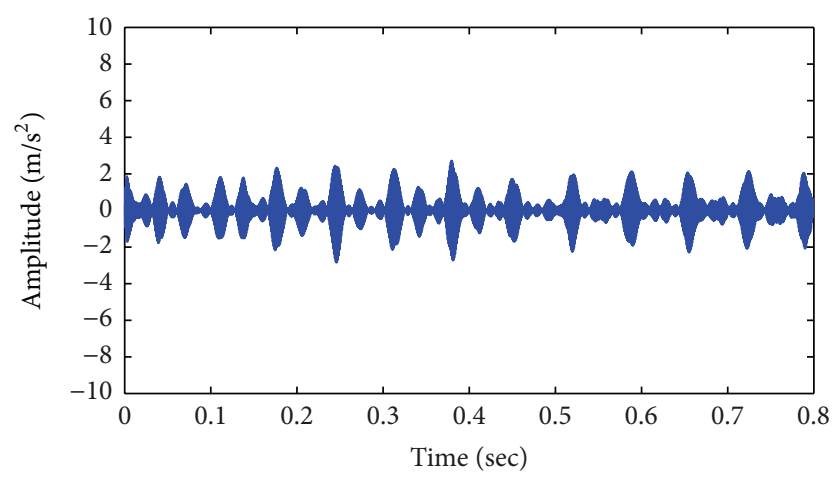

FIGURE 17: Reconstructed signal over the band 900-1200 Hz.

combining SVD with frequency slice analysis can effectively extract the shock pulses of the gears.

(2) Before frequency slice wavelet transform is performed on the vibration signal, the appropriate order to reduce noise is selected based on the differential spectrum of SVD. It is effective to remove the noise from the useful signal, and it is beneficial for the timefrequency analysis.

(3) Compared with the traditional Fourier transform, the FSWT which introduces the frequency section function is efficient for the time-frequency analysis of the

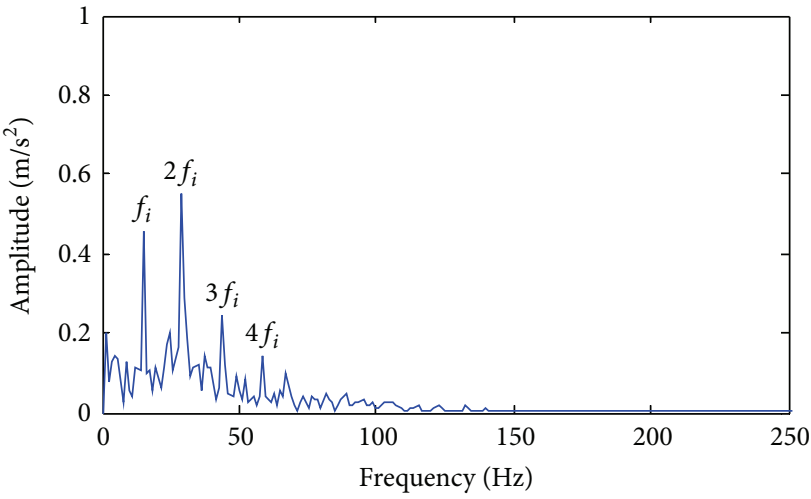

FIGURE 18: Envelope spectrum of reconstruction signal after FSWT.

signal. And the segmentation and reconstruction of any band component can be obtained. The tested gear signal analysis using the proposed method confirms the effectiveness of the method in extracting the fault features.

\section{Competing Interests}

The authors declare that there is no conflict of interests regarding the publication of this paper.

\section{Acknowledgments}

This work is supported by the National Natural Science Foundation of China (51307058), the Natural Science Foundation of Hebei Province, China (E2014502052, E2015502013), and the Chinese Fundamental Research Funds for the Central Universities (2014MS154, 2015ZD27).

\section{References}

[1] J. Rafiee, M. A. Rafiee, and P. W. Tse, "Application of mother wavelet functions for automatic gear and bearing fault diagnosis," Expert Systems with Applications, vol. 37, no. 6, pp. 45684579, 2010.

[2] F. Al-Badour, M. Sunar, and L. Cheded, "Vibration analysis of rotating machinery using time-frequency analysis and wavelet 
techniques," Mechanical Systems and Signal Processing, vol. 25, no. 6, pp. 2083-2101, 2011.

[3] Z. Yan, A. Miyamoto, and Z. Jiang, "Frequency slice wavelet transform for transient vibration response analysis," Mechanical Systems and Signal Processing, vol. 23, no. 5, pp. 1474-1489, 2009.

[4] Z. Yan, A. Miyamoto, Z. Jiang, and X. Liu, "An overall theoretical description of frequency slice wavelet transform," Mechanical Systems and Signal Processing, vol. 24, no. 2, pp. 491-507, 2010.

[5] C.-D. Duan and Q. Gao, "Noval fault diagnosis approach using time-frequency slice analysis and its application," Journal of Vibration and Shock, vol. 30, no. 9, pp. 1-5, 2011.

[6] C. Duan, Q. Gao, and X. Xu, "Generator unit fault diagnosis using the frequency slice wavelet transform time-frequency analysis method," Proceedings of the CSEE, vol. 33, no. 2, pp. 96102, 2013.

[7] X.-Y. Zhong, C.-H. Zhao, B.-J. Chen, and L.-C. Zeng, "Bearing fault diagnosis method based on morphological filtering, timedelayed autocorrelation and time-frequency slice analysis," Journal of Vibration and Shock, vol. 33, no. 4, pp. 11-16, 2014.

[8] Q. Zhu, J. Liu, and Y. Li, "Study on noisereduction in singular value decomposition based on structural risk minimization," Journal of Vibration Engineering, vol. 18, no. 2, pp. 204-207, 2005.

[9] B. Tang, Y. Jiang, and X. Zhang, "Feature extracton method of rolling bearing fault based on singular value decompositionmorphology filter and empirical mode decomposition," Journal of Mechanical Engineering, vol. 46, no. 5, pp. 37-42, 2010.

[10] J. Wang, J. Li, and X. Wan, "Fault feature extraction method of rolling bearings based on singular value decomposition and local mean decomposition," Journal of Mechanical Engineering, vol. 51, no. 3, pp. 104-110, 2015.

[11] E.-L. Chen, X. Zhang, Y.-J. Shen, and X.-M. Cao, "Fault diagnosis of rolling bearings based on SVD denoising and blind signals separation," Journal of Vibration and Shock, vol. 31, no. 23, pp. 185-190, 2012.

[12] Z. Qian, L. Cheng, and Y. Li, "Noise reduction method based on singular value decomposition," Journal of Vibration, Measurement \& Diagnosis, vol. 31, no. 4, pp. 459-463, 2011.

[13] J.-G. Wang, J. Li, and Y.-Y. Liu, "An improved method for determining effective order rank of SVD denoising," Journal of Vibration and Shock, vol. 33, no. 12, pp. 176-180, 2014. 


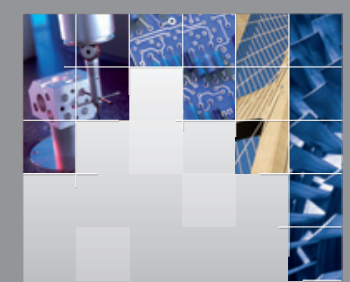

\section{Enfincering}
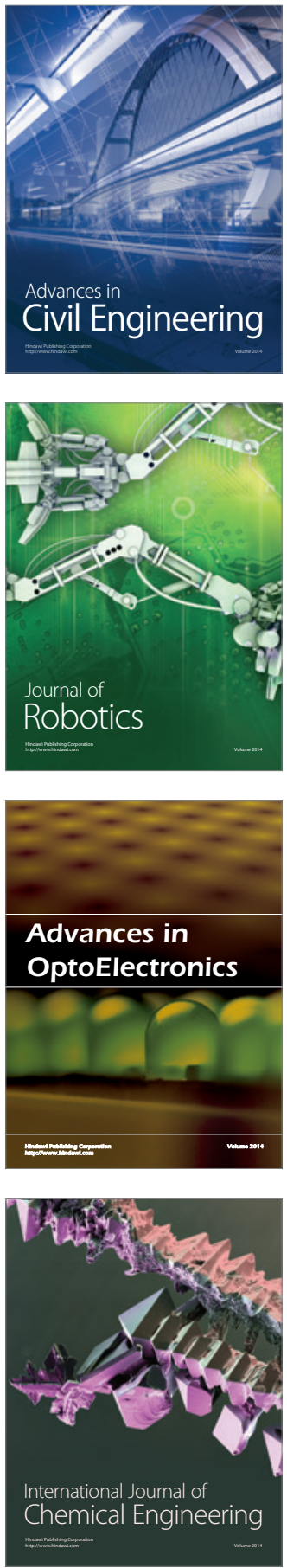

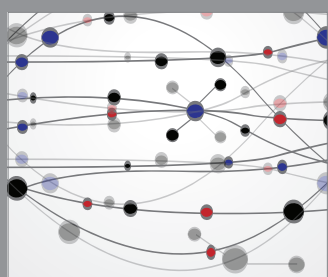

The Scientific World Journal

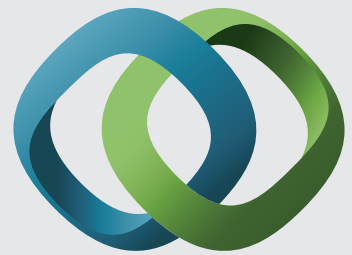

\section{Hindawi}

Submit your manuscripts at

http://www.hindawi.com
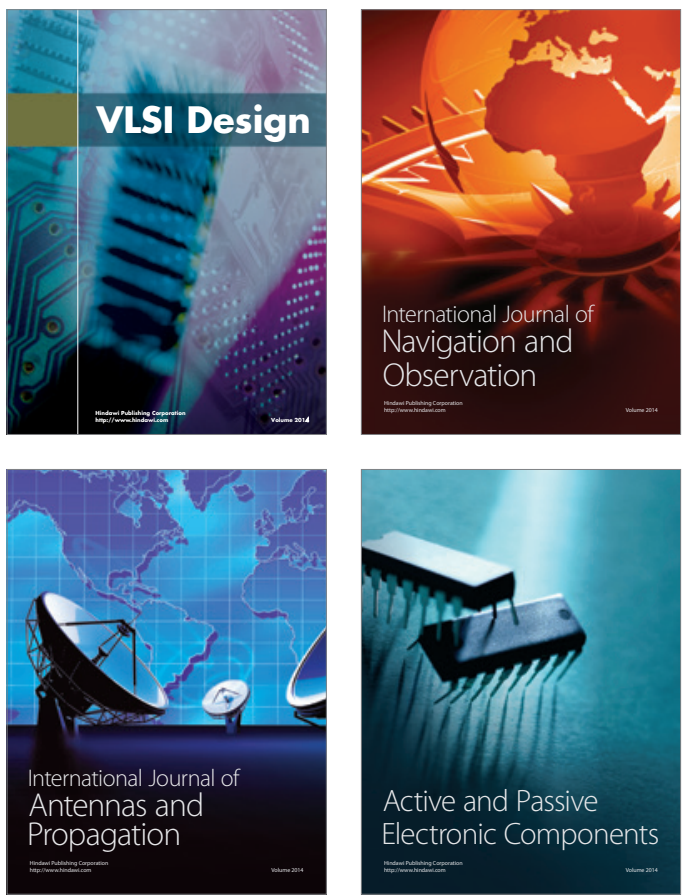
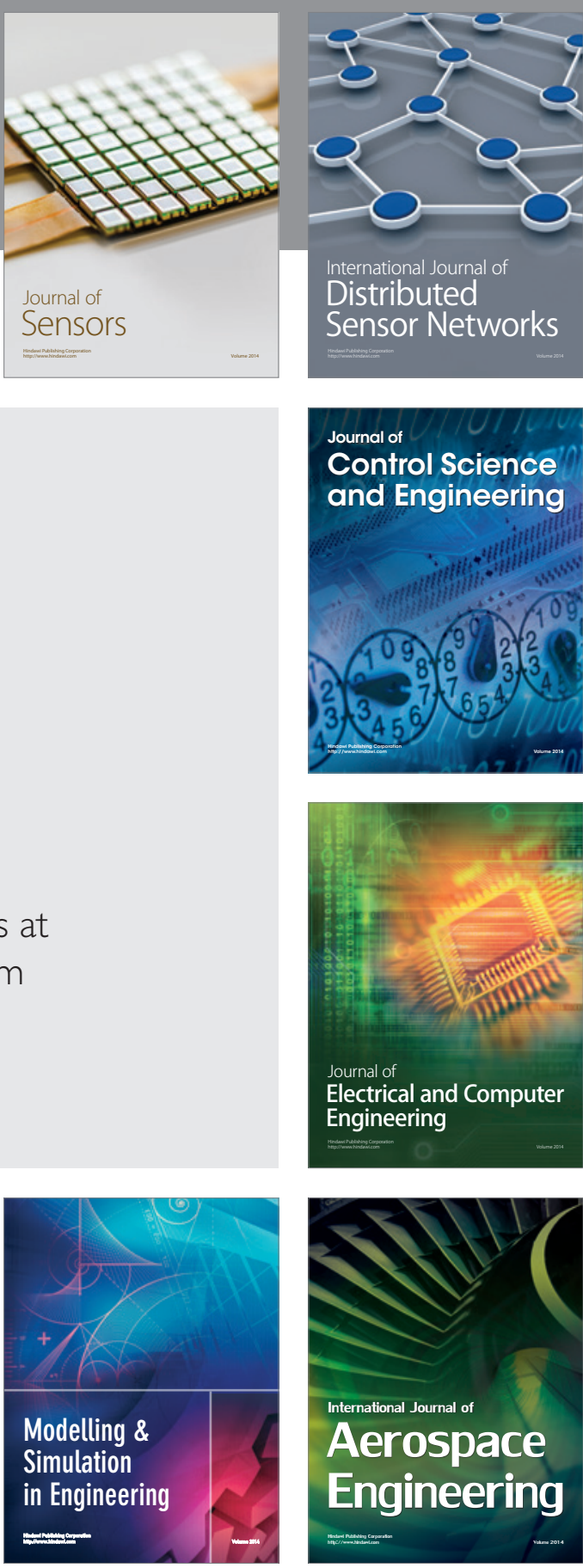

International Journal of

Distributed

Sensor Networks

Journal of

Control Science

and Engineering
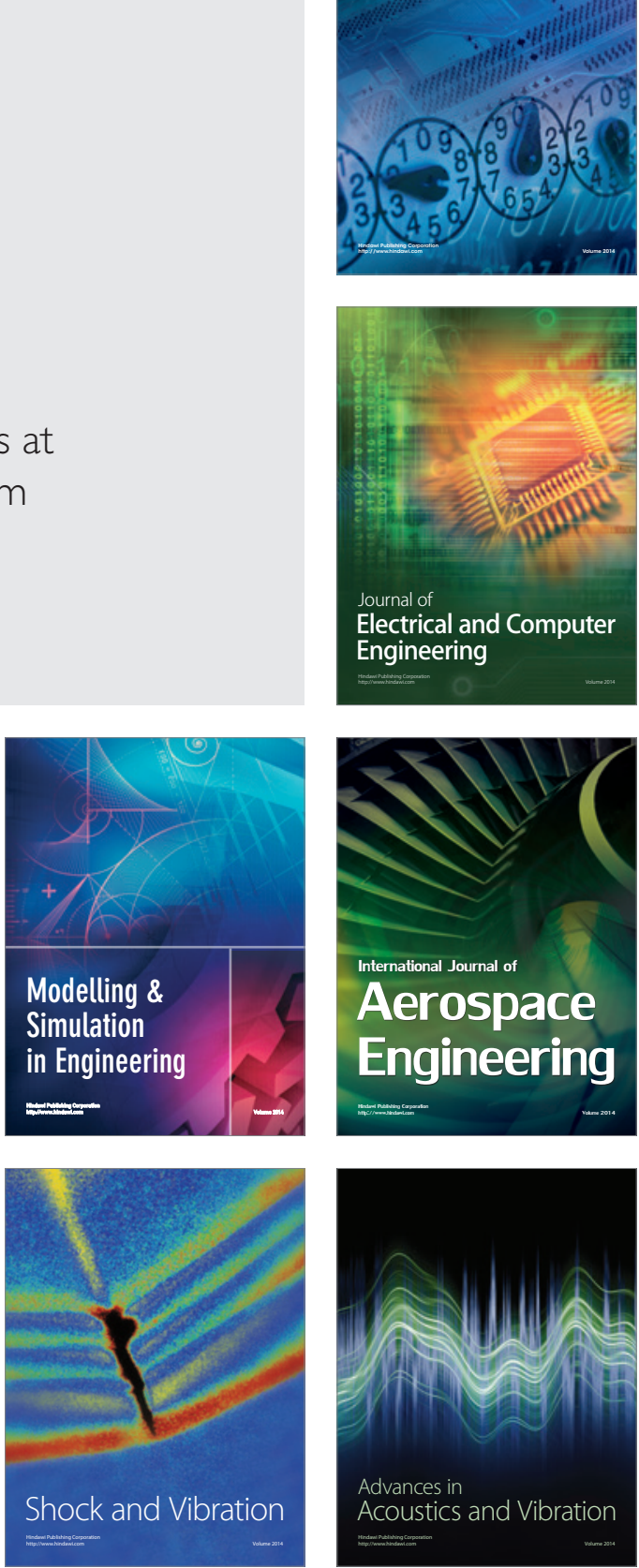